\title{
Stem Cell Therapy as a Treatment for Osteogenesis Imperfecta
}

\author{
Cecilia Götherström $^{1}$ (D) Lilian Walther-Jallow $^{1}$
}

Published online: 24 July 2020

(C) The Author(s) 2020

\begin{abstract}
Purpose of Review Osteogenesis imperfecta (OI) is a chronic disease with few treatment options available. The purpose of this review is to provide an overview on treating OI with mesenchymal stem cells (MSC).

Recent Findings Off-the-shelf MSC have a good safety profile and exhibit multilineage differentiation potential and a low immunogenic profile and are easy to manufacture. Their ability to migrate, engraft, and differentiate into bone cells, and also to act via paracrine effects on the recipient's tissues, makes MSC candidates as a clinical therapy for OI. Due to their high osteogenic potency, fetal MSC offer an even higher therapeutic potential in OI compared with MSC derived from adult sources. Preclinical and initial clinical data support the use of MSC in treating OI.

Summary The characteristics of MSC make them of great interest in treating OI. MSC may be safely transplanted via intravenous administration and show potential positive clinical effects.
\end{abstract}

Keywords Osteogenesis imperfecta $\cdot$ Brittlebones $\cdot$ Stem cell therapy $\cdot$ Stem cell transplantation $\cdot$ Prenatal therapy $\cdot$ Mesenchymal stem cells

\section{Osteogenesis Imperfecta}

One child among 10,000 to 20,000 is born with osteogenesis imperfecta (OI), a group of genetic disorders caused by $>1400$ different dominant and $>150$ recessive known mutations, and new mutations continue to be found [1,2]. Mutations in the collagen genes resulting in abnormal collagen microfibril assembly are the most common. The major clinical manifestations are atypical skeletal development, osteopenia, multiple painful fractures, and short stature, but individuals with OI may also suffer from other disorders including brittle teeth, hearing loss, and hypermobile joints. Throughout their lifetime, they also have a higher risk of pulmonary problems, heart disease (including valve insufficiency and aneurysms) that usually becomes evident only in later childhood or adulthood, and bleeding and coagulation deficiencies. Life expectancy is

This article is part of the Topical Collection on Rare Bone Diseases

Cecilia Götherström

Cecilia.Gotherstrom@ki.se

1 Department of Clinical Science, Intervention and Technology, Division of Obstetrics and Gynecology, Karolinska Institutet, ANA Futura, floor 8, Alfred Nobels Allé 8, 14152 Huddinge,

Stockholm, Sweden not affected in milder OI types but may be shortened for those with more severe types [3]. OI presents in a clinically heterogeneous manner, ranging from the mild Type I to lethal Type IIA/C [1, 2]. In 1979 OI was classified by Sillence et al. into four groups (Types I-IV) according to clinical, radiological, and histological findings $[4,5]$. Type I OI is the mildest form and may involve only a few fractures over a person's lifetime, whereas Type IIA/C OI is lethal. Type III OI is the most severe form that is compatible with survival to adulthood. Individuals affected by Type III OI may experience hundreds of fractures in a lifetime. Most commonly, OI presents in childhood with multiple fractures after little or no trauma (Type I/mild Type IV). Type IV can be moderately severe but are very variable. The growing knowledge about the genetic cause of OI have challenged the first classification by Sillence et al., and new classifications have been proposed in 2004, 2007, and 2010 [6-8]. More than 17 proteins have been described that interact directly or indirectly with the biosynthesis of collagen, and a mutation in one of these genes results in rare forms of mostly autosomal recessive OI, which share the phenotypic features of the classical OI Types I-IV. Therefore, the first classification by Sillence and colleagues has been expanded with OI Types V-VIII and includes other forms of OI based on the underlying mutations [8]. The first classification system is currently used as a grading of clinical severity of OI. 
No cure for OI exists, and current treatments do not address the underlying molecular pathology. The goal of treatment is to increase overall bone strength to prevent fractures, maintain mobility, promote normal function, and improve the quality of life. This goal is accomplished with physiotherapy to strengthen the muscles and improve mobility and lifelong orthopedic interventions such as rods in the long bones for correcting bone deformities. Bisphosphonates are used to reduce bone resorption and increase bone mineral density. The effect of bisphosphonates in OI has been debated, and according to a recent systematic review, randomized controlled trials did not demonstrate a significant improvement in function and mobility with oral bisphosphonate administration, whereas nonrandomized open-label uncontrolled studies demonstrate that oral and intravenous bisphosphonate administration objectively improved function and mobility [9].

\section{Stem Cells as a Treatment of Osteogenesis Imperfecta}

Given the severe clinical manifestations of OI and the limitations of current treatments, a clear unmet medical need exists for treatment of OI. Transplantation of mesenchymal stem cells (MSC) presents a potential new mode of treatment, specifically starting treatment before birth or as early as possible after birth, to prevent irreversible damage (see information about the BOOSTB4 trial below).

MSC are stromal cells, originally identified in adult bone marrow, and display colony-forming capacity and are nonhematopoietic and non-endothelial. They are multipotent cells that can be easily expanded and can differentiate into a variety of cell types including osteoblasts, chondrocytes, myocytes, and adipocytes [10]. In immunocompetent allogeneic/ xenogeneic animal models, intravenously infused MSC engraft widely in multiple tissues and demonstrate site-specific differentiation [11-14]. In addition, MSC constitutively secrete an extensive array of factors and microvesicles that may act in a paracrine fashion in vivo $[15,16]$.

\section{Advantages of Fetal-Derived MSC for Treatment of OI}

Fetal MSC are found at a higher frequency per nucleated cell and have a greater colony-forming capacity than adult MSC. First-trimester fetal MSC demonstrate higher proliferative capacity and have longer telomeres, a superior proliferative potential, and cycle faster than adult MSC [17]. However, fetal MSC do senesce and stop dividing within the Hayflick limit (defined as the number of times a normal human somatic cell will divide before cell division stops) but can be expanded for more population doublings than adult MSC before senescing
$[18,19]$. Apart from the mesodermal lineages, fetal MSC possess the ability to differentiate to muscle cells, and oligodendrocytes [13, 20-23], and differentiate more readily into bone than adult MSC [19, 24, 25]. Upon osteogenic differentiation, fetal MSC produced more robust osteogenic genes and induced more calcium production in vitro and reached higher levels of osteogenic gene upregulation both in vivo and in vitro than adult MSC [24]. In another direct comparison, fetal first-trimester MSC were compared with MSC derived from the term umbilical cord, adult adipose tissue, and adult bone marrow. It was shown that all MSC had equivalent immune-phenotype but that the fetal MSC exhibited the greatest osteogenic capacity [19].

In principle, allogeneic cells are subject to HLA-mediated elimination from the circulation by the host's immune system. MSC, however, have low immunogenicity, and fetal MSC are potentially even less immunogenic than adult MSC. Both fetal and adult MSC express intermediate levels of HLA class I on the surface, but adult MSC express these antigens at slightly higher levels than fetal MSC do [17, 19, 26-28]. MSC possess immunomodulatory properties demonstrated by their ability to inhibit lymphocyte proliferation, but fetal MSC are not as immunomodulatory as adult MSC and mainly inhibit mitogen-activated lymphocytes [26, 27, 29, 30]. If MSC are stimulated with IFN $\gamma$ for full expression of human leukocyte antigen (HLA) class II, the major transplantation antigen, they still escape recognition by alloreactive T cells [26, 27], and MSC differentiated to osteogenic and adipogenic lineages are also non-immunogenic [26, 27$]$.

\section{Preclinical Models on MSC Treatment of OI}

Nonclinical pharmacological studies of MSC demonstrate their potential for treatment of OI. The utility of human fetal MSC for bone regeneration was demonstrated in a rat model of critical-size bone defects treated with implant of tissueengineered bone graft seeded with fetal human MSC [25]. The MSC tissue-engineering implant group had better bone formation, more compact and woven bone, stiffer bone, and a larger vasculature network in the defect area than the control group and the group implanted with only an acellular scaffold. Studies in mouse models of OI have shown successful engraftment of human adult MSC, human fetal MSC, and mouse MSC transplanted pre- or postnatally [31-35]. The human fetal MSC were particularly detected in the bones and at sites of bone formation and remodeling and fracture healing sites, and the transplanted cells differentiated into mature osteoblasts expressing osteocalcin and producing collagen COL1a2 protein [35]. Upregulation of endogenous genes involved in early stages of endochondral and intramembranous ossification, and late hypertrophic chondrocyte differentiation was also observed [34]. Transplantation of human fetal MSC 
resulted in reduced bone brittleness with a decrease in long bone fracture rates by $67 \%$ to $84 \%$. It also lead to increased bone thickness, volume, stiffness, and strength and increased collagen and mineral content and decreased hydroxyproline content in bones $[34,35]$. Normalization of growth plate height was also observed [35].

\section{Safety and General Clinical Knowledge of MSC}

In completed and ongoing clinical trials, intravenous infusion of autologous or allogeneic MSC appears safe and well tolerated. Because of these characteristics, MSC (mostly adult) have been tested in clinical trials for a diverse variety of disorders $[36,37 \bullet \cdot, 38 \bullet \cdot$. In these trials, thousands of patients have received treatment, mostly with MSC derived from adult sources, and few adverse events have been reported. A 2012 meta-analysis of the randomized clinical trials did not detect an association with acute infusion toxicity, organ system complications, infection, death, or malignancy although a significant association between MSC and transient fever was observed after up to 90 months of follow-up [36]. The metaanalysis included both autologous and allogeneic MSC as well as expanded/cultured cells. A concern within the field has been the risk for tumor formation in the treated patients. Upon preparation for the clinical BOOSTB4 study described below, several batches of clinical grade fetal MSC were tested for and showed no tumor development in a mouse model, displayed a normal karyotype after expansion, did not express pluripotent-associated markers, and did not grow anchorageindependently. Further, the cells stopped dividing at passages 15-17 (36-48 population doublings) which is in line with the Hayflick limit for somatic cells. Hence, the risk for tumor formation can be considered to be very low. Further, no immunogenic, immunomodulatory, or tumorigenic effects relevant for clinical studies were found in preclinical animal studies.

\section{Advantages of Early Treatment of Osteogenesis Imperfecta}

\section{Early Childhood Treatment}

Initiation of treatment with MSC of severe types of OI is desirable, as early as possible before additional pathology occurs, in order to achieve the objectives outlined in Table 1 . With early treatment the rapid growth of the baby/child provides an opportunity for engraftment, expansion, and subsequent migration and distribution of the donor cells to different anatomical compartments. The effect of MSC may be enhanced with early treatment because a lesser amount of poor quality OI type collagen that requires replacement has been produced. Small numbers of donor cells result in a large amount of healthy collagen: $2 \%$ engrafted donor cells led to $20 \%$ healthy collagen in the bones in a mouse model of OI [32].

\section{Prenatal Treatment}

Fetuses affected by OI suffer from the disease even during fetal life. The natural history of the disease during fetal life is that fractures continue to develop and can be observed on ultrasound imaging as gestation advances. Thus, there is a rationale for treatment to begin as early as possible and thereby ameliorate pathology that has begun already during fetal life, or even prevent fractures from occurring. Apart from the objectives described in the first part of Table 1, additional benefits of initiation of treatment of severe types of OI during fetal life compared with postnatally are described in the second part of Table 1 .

\section{Clinical Experience of Stem Cell Treatment of Osteogenesis Imperfecta}

Postnatal stem cell transplantation for the treatment of OI has been attempted in children diagnosed with severe OI. The first clinical proof of principle came from Horwitz et al. in which six children with OI Type III were transplanted with HLAmatched whole bone marrow donated by a sibling [39]. The linear growth increased from $1.25 \mathrm{~cm}$ at 6 months before compared with $7.5 \mathrm{~cm}$ at 6 months after transplantation. During the same time period, the fracture frequency was reduced by $80 \%$ in spite of low-level $(<2 \%)$ donor osteoblast engraftment. In a follow-up trial [40], MSC were isolated from the bone marrow donors, culture expanded, and administered intravenously to the recipients. Two doses of MSC showed similar results as the bone marrow transplantation with low (1-2\%) donor cell engraftment in the bones and an acceleration of the growth velocity of $60-94 \%$ [40]. As explained above, data from mouse studies suggest that significant amounts of normal collagen can be deposited by a relatively small population of donor cells [32], which can explain the marked improvements despite low-level engraftment. No severe adverse events were noted, but one adverse event was noted in one patient, an easily treated urticarial rash after the second MSC infusion. No donor cell engraftment or clinical effect was observed in this patient.

Two case studies of prenatal transplantation of fetal MSC for the treatment of OI Type III and IV have been published $[41,42 \bullet \cdot]$. Both children subsequently received postnatal booster infusions of MSC from the same donor because of a deteriorating clinical picture. While it is difficult to definitively determine the effect of MSC transplantation on OI, the results from these two heterogeneous cases suggest that prenatal MSC 
Table 1 Objectives for early initiation of MSC treatment of severe OI

\section{Reasons for initiation of early treatment with MSC \\ Desired effect \\ Description}

Reduction of detrimental effects on respiration

Improvement in healing of fractures

Reduction of risk of additional fractures

Reduction of risk of bowing of long bones

Improvement in growth
Improve/arrest the abnormal chest wall and spine architecture (kyphoscoliosis, scoliosis, rib fractures, pectus deformities, and abnormalities of lung collagen)

Enhanced fracture healing will reduce negative effects of fractures, alleviate pain, and improve the quality of life for individuals with OI

Reduced frequency of fractures will diminish negative effects of fractures, alleviate pain, and improve the quality of life for individuals with OI

Reduced risk of bowing decreases the need for orthopedic surgical procedures and hospital stay, improves the mobility, alleviates pain, and improves the quality of life for individuals with $\mathrm{OI}$

Increased/not plateaued growth will improve the mobility and the quality of life for individuals with OI

\section{Additional reasons for initiation of prenatal treatment with MSC \\ Desired effect \\ Description}

Better physiological conditions for systemic distribution of the administered cells

Better distribution and engraftment of the cells

Better effect of engrafted cells

Naïve immune system

Psychosocial value

Improved parent and family quality of life
As the administration is given into the umbilical vein in the fetus, it will bypass the pulmonary circulation via two fetal shunts, the ductus arteriosus, and the foramen ovale. This ensures that the administered MSC go directly into the systemic circulation and can then home directly to the bones. MSC administration after birth is performed into a peripheral vein, with many MSC becoming trapped in the microcirculation of the lungs before fewer MSC reach the systemic circulation

Fetal life is a time of natural stem cell proliferation and migration to different anatomic compartments. The rapid growth of the fetus provides an opportunity for higher engraftment, wider expansion, and subsequent migration and distribution of the donor cells

The effect of MSC may be enhanced with prenatal treatment because a lesser amount of poor quality OI type collagen that requires replacement has been produced. As described above, small numbers of donor cells result in a large amount of healthy collagen

The relatively naive fetal immune system may permit the development of immune tolerance towards donor cells

A better psychosocial situation for the mother and father may result from the birth of a child who has already been treated

Having a child with chronic disease significantly affects the parents and the families quality of life, which includes both psychological and physical quality of life

Additional theoretical benefits of MSC treatment

Improvement of

Bone mineral density

Motor milestones

Quality of life
Reduction of

Platyspondyly (vertebral fractures)

Pain

Hospital stay transplantation with postnatal booster transplantation is safe and provides a potential clinical benefit, particularly in comparison with untreated individuals with the same mutation. The two cases are described in more detail below.
The first case, a Swedish patient diagnosed with OI, received one prenatal transplantation in 2002 [41]. Ultrasound examinations in gestational weeks 15 to 27 showed that all the limbs were below the fifth percentile with angulated and 
fractured femur bones. The karyotype was normal. A clinical diagnosis of severe Type III OI was made. She received a dose of $5.0 \times 10^{6}$ HLA-unmatched human first-trimester liver-derived $\mathrm{MSC} / \mathrm{kg}$ estimated fetal body weight into the intrahepatic part of the umbilical vein at gestational week 32. The procedure was well tolerated by the fetus and the pregnant woman. No fractures occurred after the MSC transplantation, and the fetus followed her growth curve, and the rest of the pregnancy was uneventful. The girl was delivered by caesarean section at gestational week 35 after spontaneous rupture of the membranes. The clinical examination after birth showed typical signs of severe OI with generalized osteopenia, platyspondyly, Wormian skull bones, thin gracile bowed long bones with deformities indicating healed fractures, and an actual fracture of the right femoral diaphysis. Bisphosphonate (pamidronate) treatment was initiated at 4 months of age due to generalized osteopenia and vertebral compression fractures.

Generally, the girl's clinical course has been better than expected from her genetic mutation. Three other individuals are known to have an identical COL1A2 mutation as this child. One child from Canada is described in Götherström et al. [42••] and presented with a very severe phenotype of OI. This patient did not receive MSC infusion and succumbed at 5 months of age due to respiratory problems despite postnatal bisphosphonates therapy. The two other individuals with the same mutation reported in the literature are described as severe OI (Type II/III). Treatment received by these individuals and other factors affecting their disease are not known. Until 8 years of age, the girl who received MSC was doing reasonably well with approximately one fracture and one compression fracture clinically confirmed every year ( 5 femoral, 2 clavicular, 1 shoulder and 1 skull fracture, and 11 vertebral compression fractures). She walked with support at 15 months of age and without support at 2 years and 4 months. Remarkably she continued growing at a normal rate, albeit around -5 SD from birth to 6 years of age, deteriorating to 6.5 SD at 8 years of age. At this point the patient received a booster dose intravenously with $2.8 \times 10^{6} / \mathrm{kg}$ same-donor MSC [42••]. This decision was made based on an increased rate of fractures and the plateaued growth. The following 2 years, the patient did not experience any new fractures, and the mobility and linear growth (-6 SD) improved. Between ages 11-13, the patient received one same-donor MSC booster dose/year to improve her height. She is now 17 years old and is doing well.

The second case was a fetus with OI Type IV who presented with short long bones ( $<$ fifth centile) and multiple new and healing fractures at 26 weeks of gestation [42••]. The fetus was transplanted with $30 \times 10^{6} / \mathrm{kg}$ HLA-unmatched human first-trimester liver-derived MSC at 31 weeks of gestation. The fetus and pregnant woman tolerated the procedure well, and the fetus did not suffer any new fractures for the remainder of the pregnancy or during infancy. Genotyping of the patient and family members identified an autosomal dominant mode of inheritance. Bisphosphonate therapy was initiated from 1 month of age due to poor mineralization. The patient followed her own growth curve just below the third percentile until 1 year of age, when the longitudinal length plateaued. A postnatal intravenous booster dose with same-donor MSC was performed at 1 year and 6 months of age, which was followed by a resumption of her lengthwise growth. She started to walk shortly after the booster dose. The girl is today 10 years old.

Donor cell engraftment has been confirmed in the first case and is limited to the bones [41, 42••]. The detected level of engraftment varies but is consistently low; between 0.003 and $16.6 \%$. Samples from the bone have not been possible to retrieve from the second case.

The two cases summarized above received intravenous doses of fetal MSC prenatally with same-donor MSC booster dose(s) postnatally. There were no signs of any early or late adverse events (follow-up more than 10 and 17 years). The donor cells did not induce any alloreactivity among the patient's lymphocytes in vitro. Before the booster doses, extensive analysis showed the absence of antibodies directed toward human leukocyte antigen class I and II, IgG and IgM, or fetal bovine serum or a cell-mediated response toward the donor MSC.

\section{A Clinical Trial Investigating MSC as a Treatment of OI}

Boost Brittle Bones Before Birth (BOOSTB4) is an European trial that investigates MSC transplantation as a therapy for severe vital forms of OI (Type III and severe Type IV). The primary endpoint of the phase I/II multicenter trial is safety and tolerability for the infant, the pregnant woman, and the fetus. Secondary endpoints relate to efficacy (fracture frequency, time to fracture, number of fractures at birth, growth, bone mineral density, biochemical bone turnover, and clinical OI status). The overall experience, impact, and perception of the therapy also will be evaluated.

The study will include three groups:

1. Four postnatal doses of $3 \times 10^{6} \mathrm{MSC} / \mathrm{kg}$ body weight $(n=15)$

2. One prenatal and three postnatal doses of $3 \times 10^{6} \mathrm{MSC} / \mathrm{kg}$ body weight $(n=15)$

3. Historical and prospective controls $(n=30-150)$

All participants who are eligible can receive MSC, i.e., there is no randomization into the groups. We have established a European network centered in Stockholm in Sweden, London in the UK, Cologne in Germany, and Utrecht/Leiden in the Netherlands. Ethical and regulatory 
approval has so far been obtained in Sweden and the UK, and the trial is open in Sweden. The BOOSTB4 consortium welcomes participants for inclusion in the clinical trial. Contact the BOOSTB4 consortium for more information: https://ki.se/ clintec and https://www.boostb4.eu.

\section{Future Outlook}

It is desirable that research groups within the cell therapy area join forces and develop common programs including guidelines regarding indications, inclusion, and manufacture of cell products. Furthermore, methods for cell transplantation, monitoring, and structured follow-up of involved fetuses and children should be collective in order for future studies to be setup with the most optimal design. If future cell therapies involving children and pregnant women and their fetuses are not performed according to the highest of standards, there is an obvious risk that the whole field will be fragmented and thus will prevent development within this area.

\section{Conclusions}

At the moment MSC transplantation before and after birth to treat $\mathrm{OI}$ is an experimental therapy about to be systematically tested in the international multicenter phase I/II clinical trial BOOSTB4 that will assess the safety and efficacy of fetal MSC transplantation for the treatment of severe types of OI.

Funding Information Open access funding provided by Karolinska Institute. This project has received funding from the European Union's Horizon 2020 research and innovation program under grant agreement 681045 and from the Swedish Research Council (E0720901).

\section{Compliance with Ethical Standards}

Conflict of Interest The authors declare that they have no conflict of interest.

Human and Animal Rights and Informed Consent This article does not contain any studies with human or animal subjects performed by any of the authors.

Open Access This article is licensed under a Creative Commons Attribution 4.0 International License, which permits use, sharing, adaptation, distribution and reproduction in any medium or format, as long as you give appropriate credit to the original author(s) and the source, provide a link to the Creative Commons licence, and indicate if changes were made. The images or other third party material in this article are included in the article's Creative Commons licence, unless indicated otherwise in a credit line to the material. If material is not included in the article's Creative Commons licence and your intended use is not permitted by statutory regulation or exceeds the permitted use, you will need to obtain permission directly from the copyright holder. To view a copy of this licence, visit http://creativecommons.org/licenses/by/4.0/.

\section{References}

Papers of particular interest, published recently, have been highlighted as:

- Of importance

- Of major importance

1. Thomas IH, DiMeglio LA. Advances in the classification and treatment of osteogenesis imperfecta. Curr Osteoporos Rep. 2016;14(1): 1-9. https://doi.org/10.1007/s11914-016-0299-y.

2. Marini JC, Forlino A, Bachinger HP, Bishop NJ, Byers PH, Paepe A, et al. Osteogenesis imperfecta. Nat Rev Dis Primers. 2017;3: 17052. https://doi.org/10.1038/nrdp.2017.52.

3. Folkestad L, Hald JD, Canudas-Romo V, Gram J, Hermann AP, Langdahl B, et al. Mortality and causes of death in patients with osteogenesis imperfecta: a register-based nationwide cohort study. $\mathrm{J}$ Bone Miner Res. 2016;31(12):2159-66. https://doi.org/10.1002/ jbmr.2895.

4. Sillence DO, Senn A, Danks DM. Genetic heterogeneity in osteogenesis imperfecta. J Med Genet. 1979;16(2):101-16.

5. Sillence DO, Horton WA, Rimoin DL. Morphologic studies in the skeletal dysplasias. Am J Pathol. 1979;96(3):813-70.

6. Rauch F, Glorieux FH. Osteogenesis imperfecta. Lancet. 2004;363(9418):1377-85.

7. Cabral WA, Chang W, Barnes AM, Weis M, Scott MA, Leikin S, et al. Prolyl 3-hydroxylase 1 deficiency causes a recessive metabolic bone disorder resembling lethal/severe osteogenesis imperfecta. Nat Genet. 2007;39(3):359-65. https://doi.org/10.1038/ng1968.

8. Van Dijk FS, Pals G, Van Rijn RR, Nikkels PG, Cobben JM. Classification of osteogenesis imperfecta revisited. Eur J Med Genet. 2010;53(1):1-5. https://doi.org/10.1016/j.ejmg.2009.10. 007.

9. Constantino CS, Krzak JJ, Fial AV, Kruger KM, Rammer JR, Radmanovic K, et al. Effect of bisphosphonates on function and mobility among children with osteogenesis imperfecta: a systematic review. JBMR Plus. 2019;3(10):e10216. https://doi.org/10.1002/ jbm4.10216.

10. Pittenger MF, Mackay AM, Beck SC, Jaiswal RK, Douglas R, Mosca JD, et al. Multilineage potential of adult human mesenchymal stem cells. Science. 1999;284(5411):143-7.

11. Liechty KW, MacKenzie TC, Shaaban AF, Radu A, Moseley AM, Deans R, et al. Human mesenchymal stem cells engraft and demonstrate site-specific differentiation after in utero transplantation in sheep. Nat Med. 2000;6(11):1282-6.

12. Devine SM, Cobbs C, Jennings M, Bartholomew A, Hoffman R. Mesenchymal stem cells distribute to a wide range of tissues following systemic infusion into nonhuman primates. Blood. 2003;101(8):2999-3001.

13. Chan J, Waddington SN, O'Donoghue K, Kurata H, Guillot PV, Götherström C, et al. Widespread distribution and muscle differentiation of human fetal mesenchymal stem cells after intrauterine transplantation in dystrophic mdx mouse. Stem Cells. 2007;25(4): 875-84. https://doi.org/10.1634/stemcells.2006-0694.

14. Guillot PV, Cook HT, Pusey CD, Fisk NM, Harten S, Moss J, et al. Transplantation of human fetal mesenchymal stem cells improves glomerulopathy in a collagen type I alpha 2-deficient mouse. J Pathol. 2008;214(5):627-36. https://doi.org/10.1002/path.2325.

15. Heldring N, Mager I, Wood MJ, Le Blanc K, Andaloussi SE. Therapeutic potential of multipotent mesenchymal stromal cells and their extracellular vesicles. Hum Gene Ther. 2015;26(8):50617. https://doi.org/10.1089/hum.2015.072.

16. Colombini A, Perucca Orfei C, Kouroupis D, Ragni E, De Luca P, Vigan OM, et al. Mesenchymal stem cells in the treatment of 
articular cartilage degeneration: new biological insights for an oldtimer cell. Cytotherapy. 2019. https://doi.org/10.1016/j.jcyt.2019. 10.004 .

17. Sagar R, Walther-Jallow L, David AL, Götherström C, Westgren M. Fetal mesenchymal stromal cells: an opportunity for prenatal cellular therapy. Curr Stem Cell Rep. 2018;4(1):61-8. https://doi. org/10.1007/s40778-018-0118-8.

18. Guillot PV, Götherström C, Chan J, Kurata H, Fisk NM. Human first-trimester fetal MSC express pluripotency markers and grow faster and have longer telomeres than adult MSC. Stem Cells. 2007;25(3):646-54. https://doi.org/10.1634/stemcells.2006-0208.

19. Zhang ZY, Teoh SH, Chong MS, Schantz JT, Fisk NM, Choolani MA, et al. Superior osteogenic capacity for bone tissue engineering of fetal compared with perinatal and adult mesenchymal stem cells. Stem Cells. 2009;27(1):126-37. https://doi.org/10.1634/stemcells. 2008-0456.

20. Chan J, O'Donoghue K, Gavina M, Torrente Y, Kennea N, Mehmet $\mathrm{H}$, et al. Galectin-1 induces skeletal muscle differentiation in human fetal mesenchymal stem cells and increases muscle regeneration. Stem Cells. 2006;24(8):1879-91. https://doi.org/10.1634/ stemcells.2005-0564.

21. Chan J, O'Donoghue K, Kennea N, de la Fuente J, Kumar S, Morgan J, et al. Myogenic potential of fetal mesenchymal stem cells. Ann Acad Med Singapore. 2003;32(5 Suppl):S11-3.

22. De Coppi P, Callegari A, Chiavegato A, Gasparotto L, Piccoli M, Taiani J, et al. Amniotic fluid and bone marrow derived mesenchymal stem cells can be converted to smooth muscle cells in the cryoinjured rat bladder and prevent compensatory hypertrophy of surviving smooth muscle cells. J Urol. 2007;177(1):369-76. https:// doi.org/10.1016/j.juro.2006.09.103.

23. Kennea NL, Waddington SN, Chan J, O'Donoghue K, Yeung D, Taylor DL, et al. Differentiation of human fetal mesenchymal stem cells into cells with an oligodendrocyte phenotype. Cell Cycle. 2009;8(7):1069-79.

24. Guillot PV, De Bari C, Dell'Accio F, Kurata H, Polak J, Fisk NM. Comparative osteogenic transcription profiling of various fetal and adult mesenchymal stem cell sources. Differentiation. 2008;76(9): 946-57. https://doi.org/10.1111/j.1432-0436.2008.00279.x.

25. Zhang ZY, Teoh SH, Chong MS, Lee ES, Tan LG, Mattar CN, et al. Neo-vascularization and bone formation mediated by fetal mesenchymal stem cell tissue-engineered bone grafts in criticalsize femoral defects. Biomaterials. 2010. https://doi.org/10.1016/j. biomaterials.2009.09.078.

26. Le Blanc K, Tammik L, Zetterberg E, Ringdén O. HLA expression and immunologic properties of differentiated and undifferentiated mesenchymal stem cells. Exp Hematol. 2003;31(10):890-6.

27. Götherström C, Ringden O, Tammik C, Zetterberg E, Westgren M, Le Blanc K. Immunologic properties of human fetal mesenchymal stem cells. Am J Obstet Gynecol. 2004;190(1):239-45.

28. Götherström C, Lundqvist A, Duprez IR, Childs R, Berg L, le Blanc $\mathrm{K}$. Fetal and adult multipotent mesenchymal stromal cells are killed by different pathways. Cytotherapy. 2011;13(3):269-78. https:// doi.org/10.3109/14653249.2010.523077.

29. Götherström C, Ringden O, Westgren M, Tammik C, Le Blanc K. Immunomodulatory effects of human foetal liver-derived mesenchymal stem cells. Bone Marrow Transplant. 2003;32(3):265-72.

30. Le Blanc K, Tammik L, Sundberg B, Haynesworth SE, Ringden O. Mesenchymal stem cells inhibit and stimulate mixed lymphocyte cultures and mitogenic responses independently of the major histocompatibility complex. Scand J Immunol. 2003;57(1):11-20.

31. Pereira RF, O'Hara MD, Laptev AV, Halford KW, Pollard MD, Class R, et al. Marrow stromal cells as a source of progenitor cells for nonhematopoietic tissues in transgenic mice with a phenotype of osteogenesis imperfecta. Proc Natl Acad Sci U S A. 1998;95(3): 1142-7.

32. Panaroni C, Gioia R, Lupi A, Besio R, Goldstein SA, Kreider J, et al. In utero transplantation of adult bone marrow decreases perinatal lethality and rescues the bone phenotype in the knockin murine model for classical, dominant osteogenesis imperfecta. Blood. 2009;114(2):459-68. https://doi.org/10.1182/blood-2008-12195859.

33. Guillot PV, Abass O, Bassett JH, Shefelbine SJ, Bou-Gharios G, Chan J, et al. Intrauterine transplantation of human fetal mesenchymal stem cells from first-trimester blood repairs bone and reduces fractures in osteogenesis imperfecta mice. Blood. 2008;111(3): 1717-25. https://doi.org/10.1182/blood-2007-08-105809.

34. Vanleene M, Saldanha Z, Cloyd KL, Jell G, Bou-Gharios G, Bassett JH, et al. Transplantation of human fetal blood stem cells in the osteogenesis imperfecta mouse leads to improvement in multiscale tissue properties. Blood. 2011;117(3):1053-60. https:// doi.org/10.1182/blood-2010-05-287565.

35. Jones GN, Moschidou D, Abdulrazzak H, Kalirai BS, Vanleene M, Osatis S, et al. Potential of human fetal chorionic stem cells for the treatment of osteogenesis imperfecta. Stem Cells Dev. 2014;23(3): 262-76. https://doi.org/10.1089/scd.2013.0132.

36. Lalu MM, McIntyre L, Pugliese C, Fergusson D, Winston BW, Marshall JC, et al. Safety of cell therapy with mesenchymal stromal cells (SafeCell): a systematic review and meta-analysis of clinical trials. PLoS One. 2012;7(10):e47559. https://doi.org/10.1371/ journal.pone.0047559.

37.• Squillaro T, Peluso G, Galderisi U. Clinical trials with mesenchymal stem cells: an update. Cell Transplant. 2016;25(5):829-48. https://doi.org/10.3727/096368915X689622. The paper summarizes the recent progress in the MSC therapy field.

38.• Nitkin CR, Bonfield TL. Concise review: mesenchymal stem cell therapy for pediatric disease: perspectives on success and potential improvements. Stem Cells Transl Med. 2017;6(2):539-65. https:// doi.org/10.5966/sctm.2015-0427. The paper summarizes the recent progress in the MSC therapy field in the pediatric population.

39. Horwitz EM, Prockop DJ, Gordon PL, Koo WW, Fitzpatrick LA, Neel MD, et al. Clinical responses to bone marrow transplantation in children with severe osteogenesis imperfecta. Blood. 2001;97(5): 1227-31.

40. Horwitz EM, Gordon PL, Koo WK, Marx JC, Neel MD, McNall $\mathrm{RY}$, et al. Isolated allogeneic bone marrow-derived mesenchymal cells engraft and stimulate growth in children with osteogenesis imperfecta: Implications for cell therapy of bone. Proc Natl Acad Sci U S A. 2002;99(13):8932-7.

41. Le Blanc K, Götherström C, Ringden O, Hassan M, McMahon R, Horwitz E, et al. Fetal mesenchymal stem-cell engraftment in bone after in utero transplantation in a patient with severe osteogenesis imperfecta. Transplantation. 2005;79(11):1607-14.

42.• Götherström C, Westgren M, Shaw SW, Åström E, Biswas A, Byers PH, et al. Pre- and postnatal transplantation of fetal mesenchymal stem cells in osteogenesis imperfecta: a two-center experience. Stem Cells Transl Med. 2014;3(2):255-64. https://doi.org/10. 5966/sctm.2013-0090. The paper describes the two first prenatal fetal MSC transplantations as treatment of $\mathrm{OI}$, which resulted in donor cell engraftment in bone and potential clinical effects.

Publisher's Note Springer Nature remains neutral with regard to jurisdictional claims in published maps and institutional affiliations. 